\title{
Smooth compactifications of certain normic bundles
}

\author{
Anthony Várilly-Alvarado • Bianca Viray
}

Received: 3 November 2014 / Revised: 29 December 2014 / Accepted: 16 January 2015 /

Published online: 20 February 2015

(C) Springer International Publishing AG 2015

Abstract For a finite cyclic Galois extension of fields $K / k$ of degree $n$ and a separable polynomial of degree $d n$ or $d n-1$, we construct an explicit smooth compactification $X \rightarrow \mathbb{P}_{k}^{1}$ of the affine normic bundle $X_{0}$ given by

$$
\mathrm{N}_{K / k}(\mathbf{z})=P(x) \neq 0,
$$

extending the map $X_{0} \rightarrow \mathbb{A}_{k}^{1}$, where $(\mathbf{z}, x) \mapsto x$. The construction makes no assumption of the characteristic of $k$, making it a suitable departure point for studying the arithmetic of smooth compactifications of $X_{0}$ over global fields of positive characteristic.

Keywords Normic bundles - Hasse principle - Smooth compactifications · Brauer-Manin obstruction

Mathematics Subject Classification $\quad 11 \mathrm{G} 35 \cdot 14 \mathrm{G} 05$

\section{Introduction}

For a finite extension $K / k$ of fields and a polynomial $P(x) \in k[x]$, the affine norm hypersurface $X_{0} \subset \mathbb{A}_{k}^{n+1}$ given by

We thank Jean-Louis Colliot-Thélène and the anonymous referee for comments improving the exposition.

A. Várilly-Alvarado $(\varangle)$

Department of Mathematics, Rice University, 6100 S. Main St. MS 136, Houston, TX 77005, USA

e-mail: av15@rice.edu

B. Viray

Department of Mathematics, University of Washington, Box 354350, Seattle, WA 98195, USA

e-mail: bviray@math.washington.edu 


$$
\mathrm{N}_{K / k}(\mathbf{z})=P(x) \neq 0
$$

parametrizes the values of $P(x)$ that are norms for $K / k$.

Suppose that $k$ is a number field. The classical Hasse norm theorem states that if $K / k$ is a cyclic Galois extension and if $P(x)$ is a nonzero constant, then $X_{0}$ satisfies the Hasse principle. Although both the Hasse principle and weak approximation fail for more general $X_{0}$, Colliot-Thélène has conjectured that the Brauer-Manin obstruction controls failures of weak approximation on any smooth proper model of $X_{0}$. See [4, Section 1] for a summary of progress towards this conjecture.

The existence of a smooth proper model $X$ of $X_{0}$ extending the projection

$$
X_{0} \rightarrow \mathbb{A}^{1}, \quad(\mathbf{z}, x) \mapsto x
$$

to a map $X \rightarrow \mathbb{P}_{k}^{1}$ is especially useful for proving arithmetic results in the direction of Colliot-Thélène's conjecture, because the map $X \rightarrow \mathbb{P}_{k}^{1}$ affords some control over the Brauer group of $X$. This map can also be used to prove that certain subsets of the number field $k$ are diophantine $[3,7,8]$.

The known constructions of $X \rightarrow \mathbb{P}^{1}$ proceed in two steps. First, one constructs a partial compactification $X^{\prime} \rightarrow \mathbb{A}_{k}^{1}$ (e.g. [2, Section 2] or [3, Proposition 2.2(i)]). Second, one extends $X^{\prime} \rightarrow \mathbb{A}_{k}^{1}$ to a map $X \rightarrow \mathbb{P}^{1}$ via Hironaka's theorem. This second step limits the scope of the construction to fields of characteristic 0 .

Our goal in this note is to give an explicit construction of a compactification $X \rightarrow \mathbb{P}_{k}^{1}$ convenient for arithmetic applications, under some hypotheses. (For example, the Picard group and Brauer group of such a compactification $X$ are easily computable; see the proofs of [8, Proposition 3.1 and Theorem 3.2].) The construction of $X$ does not impose a restriction on the characteristic of $k$; it therefore serves as a starting point for studying the arithmetic of smooth compactifications of $X_{0}$ over global fields of positive characteristic.

Theorem 1.1 Let $K / k$ be a cyclic Galois extension of fields of degree $n$, and let $P(x) \in k[x]$ be a separable polynomial of degree $d n$ or $d n-1$. There exists a smooth proper compactification $X$ of $X_{0}$, fibered over $\mathbb{P}_{k}^{1}=\operatorname{Proj} k\left[x_{0}, x_{1}\right]$, such that $X \rightarrow \mathbb{P}_{k}^{1}$ extends the map $X_{0} \rightarrow \mathbb{A}_{k}^{1}$. Furthermore, the generic fiber of $X \rightarrow \mathbb{P}_{k}^{1}$ is a SeveriBrauer variety, and the degenerate fibers lie over $V\left(P\left(x_{0} / x_{1}\right) x_{1}^{d n}\right)$, and consist of the union of $n$ rational varieties all conjugate under $\operatorname{Gal}(K / k)$.

\subsection{Outline}

Our construction of a smooth compactification takes a cue from work of Kang: the generic fiber of our construction is the embedded Severi-Brauer variety in [6].

In Sect. 3.2, we construct a partial compactification $Y_{a} \rightarrow \operatorname{Spec} R$ of the variety $z_{1} \cdots z_{n}=a \neq 0$ for any $k$-algebra $R$ with no zero-divisors and any $a \in R \backslash 0$. In Sect. 3.3, we give an explicit open covering of $Y_{a}$, which we use in Sect. 3.4 to prove that $Y$ is smooth if and only if $V(a) \subset \operatorname{Spec} R$ is smooth. We describe the geometry of the degenerate fibers of $Y_{a} \rightarrow$ Spec $R$ in Sect. 3.5. 
In Sect. 4, we construct a $K / k$-twist of $Y_{a}, X_{K, a}^{0} \rightarrow$ Spec $R$. Finally, in Sect. 5, we restrict to the case $R=k[x]$ and $a=P(x)$, give a full compactification $X \rightarrow \mathbb{P}_{k}^{1}$, and prove Theorem 1.1.

Remark 1.2 Let $R$ be a Dedekind domain, and let $\mathcal{O}$ be a maximal $R$-order in a central simple Frac $(R)$-algebra of rank $n^{2}$. Write $X_{\mathcal{O}} \rightarrow \operatorname{Spec} R$ for the scheme of rank $n$ left ideals of $\mathcal{O}$. In [1] Artin studies the geometry of $X_{\mathcal{O}} \rightarrow$ Spec $R$ through its functor of points. By taking $R=k[x]$, and an appropriate choice of $\mathcal{O}$, this gives a variety $X_{\mathcal{O}}$ proper over $\mathbb{A}_{k}^{1}$, whose generic fiber is the Severi-Brauer variety associated to $X_{0, k(x)}$, and whose degenerate fibers consist of $n$ rational varieties conjugate under $\operatorname{Gal}(K / k)$.

The functor of points description of $X_{\mathcal{O}}$ highlights the connection with central simple algebras, but the embedding $X_{0, k(x)} \hookrightarrow X_{\mathcal{O}}$ is not easily extracted from this description. In contrast, our construction of a compactification of $X_{0}$ takes the norm form $X_{0}$ as a starting point, and thus the embedding $X_{0} \hookrightarrow X$ is readily apparent in the construction.

\section{Preliminaries on vectors}

Throughout, we fix an integer $n>1$. By the weight of a vector $\mathbf{v}=\left(v_{m}\right)_{m=0}^{n-1} \in \mathbb{Z}^{n}$, we mean the sum $\sum_{m=0}^{n-1} v_{m}$; we also say $\mathbf{v}$ has length $n$. Let $\mathcal{V}_{n}$ denote the set of nonnegative integer vectors of weight $n$ and length $n$. Write $\sigma: \mathcal{V}_{n} \rightarrow \mathcal{V}_{n}$ for the shift operator

$$
\mathbf{v}=\left(v_{0}, v_{1}, \ldots, v_{n-1}\right) \mapsto\left(v_{1}, v_{2}, \ldots, v_{n-1}, v_{0}\right)=\sigma(\mathbf{v})
$$

For any $\mathbf{v}=\left(v_{m}\right)_{m=0}^{n-1} \in \mathcal{V}_{n}$ we define two nonnegative integers:

$$
\begin{aligned}
& \mu(\mathbf{v})=\max _{i \in(0, n]}\left(i-v_{0}-\cdots-v_{i-1}\right), \\
& \lambda(\mathbf{v})=\mu(\mathbf{v})+\mu(\sigma(\mathbf{v}))+\cdots+\mu\left(\sigma^{n-1}(\mathbf{v})\right),
\end{aligned}
$$

and for any integers $i, j$ with $i \not \equiv j \bmod n$ and $v_{j}>0$, we let $\mathbf{v}^{i j}=\left(v_{m}+\delta_{i \bmod n, m}-\right.$ $\left.\delta_{j \bmod n, m}\right)_{m=0}^{n-1}$; note that $\mathbf{v}^{i j} \in \mathcal{V}_{n}$, because $v_{j}>0$. We collect a few straightforward relations used frequently below.

Lemma 2.1 We have the following relations.

(a) $\mu\left(\sigma^{s}(\mathbf{v})\right)=\mu(\mathbf{v})+v_{0}+v_{1}+\cdots+v_{s-1}-s$ for any $\mathbf{v} \in \mathcal{V}_{n}$ and for any $s \in(0, n]$.

(b) For any integer $r$ and any vectors $\mathbf{v}_{i}, \mathbf{w}_{i} \in \mathcal{V}_{n}$ with $1 \leq i \leq r$, such that $\sum_{i=1}^{r} \mathbf{w}_{i}=$ $\sum_{i=1}^{r} \mathbf{v}_{i}$, we have $\sum_{i=1}^{r} \lambda\left(\mathbf{w}_{i}\right)-\sum_{i=1}^{r} \lambda\left(\mathbf{v}_{i}\right)=n\left(\sum_{i=1}^{r} \mu\left(\mathbf{w}_{i}\right)-\sum_{i=1}^{r} \mu\left(\mathbf{v}_{i}\right)\right)$.

(c) Fix integers $0 \leq r<s<n$ and fix $\mathbf{v}=\left(v_{m}\right)_{m=0}^{n-1} \in \mathcal{V}_{n}$ such that $v_{r}, v_{s}>0$. Then $0 \leq \mu\left(\mathbf{v}^{r s}\right)+\mu\left(\mathbf{v}^{s r}\right)-2 \mu(\mathbf{v}) \leq 1$, and the first inequality is strict if and only if $\mu(\mathbf{v})=i-\sum_{m=0}^{i-1} v_{m}=j-\sum_{m=0}^{j-1} v_{m}$ for some $i \in(r, s]$ and $j \in(0, r] \cup(s, n]$.

Proof Let $\mathbf{w}=\sigma^{s}(\mathbf{v})$, so that $w_{j}=v_{j+s}$ if $j<n-s$ and $w_{j}=v_{j+s-n}$ if $j \geq n-s$. Then $i-w_{0}-w_{1}-\cdots-w_{i-1}$ equals 
$\begin{cases}\left((i+s)-v_{0}-\cdots-v_{i+s-1}\right)+v_{0}+\cdots+v_{s-1}-s & \text { if } i+s \leq n \\ \left((i+s-n)-v_{0}-\cdots-v_{i+s-n-1}\right)+(n-s)-v_{s}-\cdots-v_{n-1} & \text { otherwise. }\end{cases}$

To conclude (a), note that since $\mathbf{v}$ has weight $n$, we have $v_{0}+v_{1}+\cdots+v_{s-1}-s=$ $(n-s)-v_{s}-v_{s+1}-\cdots-v_{n-1}$.

By (a), for any vector $\mathbf{v}=\left(v_{m}\right)_{m=0}^{n-1} \in \mathcal{V}_{n}$ we have

$$
\lambda(\mathbf{v})=n \mu(\mathbf{v})+\sum_{m=0}^{n-1}\left((n-1-m) v_{m}-m\right) .
$$

Using the assumption that $\sum_{i=1}^{r} \mathbf{w}_{i}=\sum_{i=1}^{r} \mathbf{v}_{i}$, the proof of (b) is now a simple manipulation.

It remains to prove (c). Let $\mathbf{w}^{-}=\mathbf{v}^{r s}$ and $\mathbf{w}^{+}=\mathbf{v}^{s r}$. Since $r<s$, by the definition of $\mu$ we have

$$
\mu(\mathbf{v})-1 \leq \mu\left(\mathbf{w}^{-}\right) \leq \mu(\mathbf{v}) \quad \text { and } \quad \mu(\mathbf{v}) \leq \mu\left(\mathbf{w}^{+}\right) \leq \mu(\mathbf{v})+1 .
$$

Furthermore, we have $\mu\left(\mathbf{w}^{-}\right)=\mu(\mathbf{v})-1$ if and only if the maximum of $\left\{i-v_{0}-\cdots\right.$ $\left.-v_{i-1}\right\}_{i \in(0, n]}$ is only achieved for $i \in(r, s]$. Similarly, we have $\mu(\mathbf{v})=\mu\left(\mathbf{w}^{+}\right)$if and only if the maximum of $\left\{i-v_{0}-\cdots-v_{i-1}\right\}_{i \in(0, n]}$ is only achieved for $i \in$ $(0, r] \cup(s, n]$.

The following notion is the fundamental book-keeping device in the construction of $X \rightarrow \mathbb{P}_{k}^{1}$.

Definition 2.2 A vector $\mathbf{v}=\left(v_{0}, v_{1}, \ldots, v_{n-1}\right) \in \mathcal{V}_{n}$ is well-spaced if

$$
v_{i}>0 \Rightarrow v_{i+v_{i}}>0 \quad \text { and } \quad v_{i+j}=0, \quad j \in\left[1, v_{i}-1\right]
$$

for all $i \in[0, n)$. Here indices are considered modulo $n$.

For example, the vector $\mathbf{v}=(0,3,0,0,2,0,4,0,0)$ is well-spaced whereas $\mathbf{w}=$ $(0,3,0,0,2,4,0,0,0)$ is not. Note that $\sigma(\mathbf{v})$ is well-spaced if and only if $\mathbf{v}$ is wellspaced.

Remark 2.3 We are unaware if well-spaced vectors arise naturally in other fields. It would be interesting to have a conceptual understanding of why these vectors yield useful affine coverings of the varieties under consideration, see Sect. 3.

Lemma 2.4 Let $\mathbf{v} \in \mathcal{V}_{n}$ be a well-spaced vector with $\ell+1$ nonzero entries indexed by $i_{0}<\cdots<i_{\ell}$. Set $i_{\ell+1}=n+i_{0}$. Then $\mu(\mathbf{v})=i_{0}$, and for any $r, s \in[0, \ell]$ and $j \in\left(i_{r}, i_{r+1}\right)$, we have

$$
\mu\left(\mathbf{v}^{j i_{r}}\right)=\mu(\mathbf{v})-\left\lfloor\frac{j}{n}\right\rfloor, \quad \mu\left(\sigma^{i_{s}}\left(\mathbf{v}^{j i_{r}}\right)\right)=0
$$


and if $\ell \neq 0$,

$$
\mu\left(\mathbf{v}^{i_{r} i_{r+1}}\right)=\mu(\mathbf{v})+\left\lfloor\frac{i_{r+1}}{n}\right\rfloor, \quad \mu\left(\sigma^{i_{s}}\left(\mathbf{v}^{i_{r} i_{r+1}}\right)\right)=\delta_{s, r+1 \bmod \ell+1} .
$$

Proof For any vector $\mathbf{v}=\left(v_{m}\right)_{m=0}^{n-1} \in \mathcal{V}_{n}$, the maximum of $\left\{i-v_{0}-\cdots-v_{i-1}\right\}_{i \in(0, n]}$ is never achieved at $i=j$ where $v_{j}=0$. Additionally, since $\mathbf{v}$ is well-spaced, $v_{i_{r}}=i_{r+1}-i_{r}$ for all $r \in[0, \ell]$. Hence

$$
\mu(\mathbf{v})=\max _{r \in[0, \ell]}\left(i_{r}-v_{i_{0}}-\cdots-v_{i_{r-1}}\right)=\max _{r \in[0, \ell]}\left(i_{r}-\left(i_{1}-i_{0}\right)-\cdots-\left(i_{r}-i_{r-1}\right)\right)=i_{0}
$$

and the maximum of $\left\{i-v_{0}-\cdots-v_{i-1}\right\}_{i \in(0, n]}$ is achieved at $i=i_{r}$ for all $r \in[0, \ell]$.

Thus, the formulas for $\mu\left(\mathbf{v}^{j i_{r}}\right)$ and $\mu\left(\mathbf{v}^{i_{r} i_{r+1}}\right)$ follow from the same argument as in Lemma 2.1 (c). Let $\mathbf{w}=\mathbf{v}^{j i_{r}}$. Then

$$
\begin{aligned}
\mu\left(\sigma^{i_{s}}(\mathbf{w})\right) & =\mu(\mathbf{w})+w_{0}+\cdots+w_{i_{s}-1}-i_{s} & & \text { by Lemma 2.1 (a), } \\
& =\mu(\mathbf{v})-\left\lfloor\frac{j}{n}\right\rfloor+w_{0}+\cdots+w_{i_{s}-1}-i_{s} & & \text { by the formula for } \mu\left(\mathbf{v}^{j i_{r}}\right), \\
& =\mu(\mathbf{v})-\left\lfloor\frac{j}{n}\right\rfloor+v_{0}+\cdots+v_{i_{s}-1}-i_{s}+\left\lfloor\frac{j}{n}\right\rfloor & & \text { by the definition of } \mathbf{v}^{j i_{r}}, \\
& =\mu(\mathbf{v})-i_{0} & & \text { since } v_{i_{r}}=i_{r+1}-i_{r} \text { for } r \in[0, \ell] .
\end{aligned}
$$

Similarly, if $\mathbf{w}=\mathbf{v}^{i_{r} i_{r+1}}$, we have

$$
\begin{aligned}
\mu\left(\sigma^{i_{s}}(\mathbf{w})\right) & =\mu(\mathbf{w})+w_{0}+\cdots+w_{i_{s}-1}-i_{s} \\
& =\mu(\mathbf{v})+\left\lfloor\frac{i_{r+1}}{n}\right\rfloor+w_{0}+\cdots+w_{i_{s}-1}-i_{s} \\
& =\mu(\mathbf{v})+\left\lfloor\frac{i_{r+1}}{n}\right\rfloor+v_{0}+\cdots+v_{i_{s}-1}-i_{s}+\delta_{s, r+1 \bmod \ell+1}-\left\lfloor\frac{i_{r+1}}{n}\right\rfloor \\
& =\mu(\mathbf{v})-i_{0}+\delta_{s, r+1 \bmod \ell+1 .}
\end{aligned}
$$

\section{The auxiliary bundle $Y \rightarrow \operatorname{Spec} R$}

\subsection{Notation}

Let $R$ be a $k$-algebra with no zero-divisors. Given a nonzero element $a \in R$, we use the standard notation $D(a)$ to denote the open affine subscheme of Spec $R$ given by Spec $R_{a}$; if $R$ is graded, we let $D_{+}(a)$ denote the open affine subscheme of Proj $R$ given by $\operatorname{Spec}\left(R_{a}\right)_{0}$.

Let $N=\left(\begin{array}{c}2 n-1 \\ n\end{array}\right)-1$, and fix coordinates on $\mathbb{P}_{k}^{N}=\operatorname{Proj} k\left[\left\{y_{\mathbf{v}}: \mathbf{v} \in \mathcal{V}_{n}\right\}\right]$. We set $\mathbb{P}_{R}^{N}=\mathbb{P}_{k}^{N} \times$ Spec $k$ Spec $R$. 


\subsection{Construction of $Y_{a}$}

For any nonzero $a \in R$, we consider the embedding

$$
\iota_{a}: \operatorname{Proj} R\left[t_{0}, \ldots, t_{n-1}\right] \cap D(a) \hookrightarrow \mathbb{P}_{R}^{N} \times \operatorname{Spec} R D(a)
$$

induced by the map $y_{\mathbf{v}} \mapsto a^{\mu(\mathbf{v})} t_{0}^{v_{0}} t_{1}^{v_{1}} \cdots t_{n-1}^{v_{n-1}}$. (This is easily seen to be an embedding since it is the composition of the $(n)$-uple embedding with a scaling of the coordinates by an appropriate power of $a$.) The image of $\iota_{a}$ is cut out by the equations, see [5, Example 2.6]:

$$
a^{\sum_{i=1}^{r} \mu\left(\mathbf{w}_{i}\right)} \prod_{i=1}^{r} y_{\mathbf{v}_{i}}=a^{\sum_{i=1}^{r} \mu\left(\mathbf{v}_{i}\right)} \prod_{i=1}^{r} y_{\mathbf{w}_{i}}
$$

for all integers $r$ and all sets of vectors $\mathbf{w}_{i}, \mathbf{v}_{i}$, with $1 \leq i \leq r$, such that $\sum_{i=1}^{r} \mathbf{w}_{i}=$ $\sum_{i=1}^{r} \mathbf{v}_{i}$. Let $Y_{a}$ be the closure in $\mathbb{P}_{R}^{N}$ of the image of $\iota_{a}$.

Lemma 3.1 The order $n$ automorphism $\phi: \mathbb{P}_{R}^{N} \rightarrow \mathbb{P}_{R}^{N}, y_{\mathbf{v}} \mapsto y_{\sigma(\mathbf{v})}$ preserves $Y_{a}$.

Proof Fix an integer $r$ and vectors $\mathbf{v}_{i}, \mathbf{w}_{i}$, with $1 \leq i \leq r$, such that $\sum_{i=1}^{r} \mathbf{v}_{i}=$ $\sum_{i=1}^{r} \mathbf{w}_{i}$. It is clear that $\sum_{i=1}^{r} \sigma\left(\mathbf{v}_{i}\right)=\sum_{i=1}^{r} \sigma\left(\mathbf{w}_{i}\right)$. Moreover, by Lemma 2.1 (a),

$$
\sum_{i=1}^{r} \mu\left(\sigma\left(\mathbf{v}_{i}\right)\right)-\sum_{i=1}^{r} \mu\left(\sigma\left(\mathbf{w}_{i}\right)\right)=\sum_{i=1}^{r} \mu\left(\mathbf{v}_{i}\right)-\sum_{i=1}^{r} \mu\left(\mathbf{w}_{i}\right) .
$$

Therefore, $\phi$ preserves the relations (1).

\subsection{An open covering}

Proposition 3.2 The open subvarieties $\left\{D_{+}\left(y_{\mathbf{v}}\right) \subseteq \mathbb{P}_{R}^{N}: \mathbf{v} \in \mathcal{V}_{n}\right.$ well-spaced $\}$ cover $Y_{a}$.

Proof Let $\mathbf{w}=\left(w_{0}, w_{1}, \ldots, w_{n-1}\right) \in \mathcal{V}_{n}$ be a vector that is not well-spaced. We will show that $D_{+}\left(y_{\mathbf{w}}\right) \cap Y_{a} \subset D_{+}\left(y_{\mathbf{v}}\right)$ for some well-spaced vector $\mathbf{v} \in \mathcal{V}_{n}$. Let $i_{0}<\cdots<i_{\ell}$ be the indices such that $w_{i_{j}}>0$, and set $i_{\ell+1}=i_{0}+n$.

If $\mu(\mathbf{w})>i_{0} \geq 0$, then there exists $r \in[0, \ell)$ such that $\mu(\mathbf{w})=i_{r+1}-w_{i_{0}}-$ $w_{i_{1}}-\cdots-w_{i_{r}}$. Fix the smallest such $r$; then $i_{r+1}-i_{r}-w_{i_{r}}>0$. Since $\mathbf{w}$ has length $n$ and weight $n$, there exists $r<s \leq \ell$ such that $i_{s+1}-i_{s}-w_{i_{s}}<0$; fix the largest such $s$. Then by our choice of $r$ and $s$, if $\mu(\mathbf{w})=j-v_{0}-\cdots-v_{j-1}$, we must have $j \in\left(i_{r}, i_{s}\right]$. Therefore by Lemma 2.1 (c), the defining equations for $Y_{a}$ include the relation

$$
y_{\mathbf{W}}^{2}=y_{\mathbf{w}^{i_{r}} i_{s}} y_{\mathbf{w}^{i_{s} i_{r}},}
$$

so $D_{+}\left(y_{\mathbf{w}}\right) \subset D_{+}\left(y_{\mathbf{w}^{i} i_{s}}\right)$. After possibly repeating the argument we may assume that $\mu(\mathbf{w})=i_{0}$. 
If $i_{r+1}-i_{r}-w_{i_{r}}=0$ for all $r$, then $\mathbf{w}$ is well-spaced. Otherwise, fix the smallest integer $r$ such that $\left|i_{r+1}-i_{r}-w_{i_{r}}\right|>0$; since $\mu(\mathbf{w})=i_{0}$, we must have $i_{r+1}-$ $i_{r}-w_{i_{r}}<0$. Since $\mathbf{w}$ has length $n$ and weight $n$, there exists $r<s \leq \ell$ such that $i_{s+1}-i_{s}-w_{i_{s}}>0$; fix the smallest such $s$. Now by our choice of $r$ and $s$, if $\mu(\mathbf{w})=j-v_{0}-\cdots-v_{j-1}$, we must have $j \in\left[0, i_{r}\right] \cup\left(i_{s}, n\right)$. Then by the same argument as above, the defining equations for $Y_{a}$ include the relation

$$
y_{\mathbf{w}}^{2}=y_{\mathbf{w}^{i_{r}} i_{s}} y_{\mathbf{w}^{i_{s}} i_{r}} .
$$

By replacing $\mathbf{w}$ with $\mathbf{w}^{i_{s} i_{r}}$, we reduce the value of $\left|i_{r+1}-i_{r}-w_{i_{r}}\right|$. Repeating this process we will arrive at a well-spaced vector $\mathbf{v}$ in finitely many steps.

\subsection{Smoothness of $Y_{a}$}

Proposition 3.3 Let $\mathbb{A}_{R}^{n}=\operatorname{Spec} R\left[Z_{0}, \ldots, Z_{n-1}\right]$. Let $\mathbf{v} \in \mathcal{V}_{n}$ be a well-spaced vector with $\ell+1$ nonzero entries indexed by $i_{0}<\cdots<i_{\ell}$ and set $i_{\ell+1}=i_{0}+n$. Then the map

$$
\frac{y_{\mathbf{w}}}{y_{\mathbf{v}}} \mapsto \prod_{\substack{j \in[0, n) \\ v_{j}=0}} Z_{j}^{w_{j}} \times \prod_{r=0}^{\ell} Z_{i_{r}}^{\mu\left(\sigma^{i_{r}+1}(\mathbf{w})\right)}
$$

yields an isomorphism $Y_{a} \cap D_{+}\left(y_{\mathbf{v}}\right) \cong V\left(Z_{i_{0}} \cdots Z_{i_{\ell}}-a\right) \subset \mathbb{A}_{R}^{n}$. In particular, $Y_{a}$ is a compactification of the variety in $\mathbb{A}_{R}^{n}$ given by $Z_{0} Z_{1} \cdots Z_{n-1}=a$.

From Propositions 3.2 and 3.3, and the Jacobian criterion we have

Corollary 3.4 The variety $Y_{a}$ is smooth if and only if $V(a)$ is smooth in $\operatorname{Spec} R$.

Proof of Proposition 3.3 Set $i_{-1}=i_{\ell}-n$. The proof of the proposition differs slightly in the case when $\ell=0$. To give a unified presentation, if $\ell=0$ then we set $y_{\mathbf{v}} i_{0} i_{\ell+1}=$ $a y_{\mathbf{v}}$. Consider the following functions on $Y_{a} \cap D_{+}\left(y_{\mathbf{v}}\right)$ for $j=i_{0}, i_{0}+1, \ldots, i_{0}+n-1$ :

$$
g_{j}= \begin{cases}y_{\mathbf{v}} j i_{r} y_{\mathbf{v}}^{-1} & \text { if } i_{r}<j<i_{r+1} \text { for some } 0 \leq r \leq \ell \\ y_{\mathbf{v}^{i} i_{r+1}} y_{\mathbf{v}}^{-1} & \text { if } j=i_{r}, 0 \leq r \leq \ell\end{cases}
$$

Lemma 2.4 shows that the map sends $g_{j} \rightarrow Z_{j \bmod n}$. In addition, Lemma 2.4 together with the relations (1) shows that $g_{i_{0}} \cdots g_{i_{\ell}}=a$. Thus, to prove the map is a welldefined isomorphism, we will show that

$$
y_{\mathbf{w}} y_{\mathbf{v}}^{m}=\prod_{\substack{j \in[0, n) \\ v_{j}=0}} y_{\mathbf{v}_{j} i_{r}}^{w_{j}} \times \prod_{r=0}^{\ell} y_{\mathbf{v}^{i_{r} i_{r+1}}}^{\mu\left(\sigma^{i_{r}+1}(\mathbf{w})\right)}
$$

where $m=-1+\sum_{j, v_{j}=0} w_{j}+\sum_{r=0}^{\ell} \mu\left(\sigma^{i_{r+1}}(\mathbf{w})\right)$. By Lemmas 2.1 (a) and 2.4, we have 


$$
\begin{aligned}
\sum_{\substack{j \in[0, n) \\
v_{j}=0}} w_{j} \cdot \mu\left(\mathbf{v}^{j i_{r}}\right) & +\sum_{r=0}^{\ell} \mu\left(\sigma^{i_{r+1}}(\mathbf{w})\right) \mu\left(\mathbf{v}^{i_{r} i_{r+1}}\right) \\
& =(m+1) \mu(\mathbf{v})-\sum_{j=0}^{i_{0}-1} w_{j}+\mu\left(\sigma^{i_{\ell+1}}(\mathbf{w})\right) \\
& =(m+1) \mu(\mathbf{v})+\mu(\mathbf{w})-i_{0}=m \mu(\mathbf{v})+\mu(\mathbf{w}) .
\end{aligned}
$$

Hence, by (1), it suffices to prove that $\mathbf{w}+m \mathbf{v}$ is equal to

$$
\mathbf{w}^{\prime}=\sum_{\substack{j \in[0, n) \\ v_{j}=0}} w_{j} \mathbf{v}^{j i_{r}}+\sum_{r=0}^{\ell} \mu\left(\sigma^{i_{r+1}}(\mathbf{w})\right) \mathbf{v}^{i_{r} i_{r+1}} .
$$

For $j$ such that $v_{j}=0$, it is evident that $w_{j}^{\prime}=w_{j}+m v_{j}$. Further,

$$
\begin{aligned}
& w_{i_{s}}^{\prime}=(m+1) v_{i_{s}}-\sum_{j=i_{s}+1}^{i_{s+1}-1} w_{j}+\mu\left(\sigma^{i_{s+1}}(\mathbf{w})\right)-\mu\left(\sigma^{i_{s}}(\mathbf{w})\right) \\
& =(m+1) v_{i_{s}}-\sum_{j=i_{s}+1}^{i_{s+1}-1} w_{j}+\sum_{j=i_{s}}^{i_{s+1}-1} w_{j}-i_{s+1}+i_{s} \quad \text { by Lemma 2.1 (a) } \\
& =m v_{i_{s}}+w_{i_{s}} \quad \text { since } v_{i_{s}}=i_{s+1}-i_{s} \text {. }
\end{aligned}
$$

\subsection{The degenerate fibers of $Y_{a} \rightarrow$ Spec $R$}

Proposition 3.5 Let $Q \in V(a) \in R$ be a closed point. The fiber $Y_{a, Q}$ consists of $n$ rational $(n-1)$-dimensional irreducible components which are permuted cyclically by the automorphism $\phi$ of Lemma 3.1.

Proof For $i=0, \ldots, n-1$, we define $S_{i}=Y_{a, Q} \cap V\left(\left\langle y_{\mathbf{w}}: \mu\left(\sigma^{i+1}(\mathbf{w})\right)>0\right\rangle\right)$. From the definition, it follows that $\phi$ acts on the set $\left\{S_{i}: 0 \leq i \leq n-1\right\}$ via the permutation

$$
S_{0} \mapsto S_{n-1} \mapsto S_{n-2} \mapsto \cdots \mapsto S_{1} \mapsto S_{0}
$$

We claim that $Y_{a, Q}=S_{0} \cup S_{1} \cup \cdots \cup S_{n-1}$ and that each $S_{i}$ is an irreducible rational $(n-1)$-dimensional variety.

Let $\mathbf{v} \in \mathcal{V}_{n}$ be a well-spaced vector and let $i_{0}<\cdots<i_{\ell}$ be the indices of the nonzero entries of v. By Proposition 3.3, $Y_{a, Q} \cap D_{+}\left(y_{\mathbf{v}}\right)$ is isomorphic to a union of $\ell+1$ hyperplanes in $\mathbb{A}_{\mathbf{k}(Q)}^{n}=\operatorname{Spec} \mathbf{k}(Q)\left[Z_{0}, \ldots, Z_{n-1}\right]$. Furthermore, the hyperplane $Z_{i_{r}}=0$ is isomorphic to the subvariety 


$$
V\left(\left\langle\frac{y_{\mathbf{w}}}{y_{\mathbf{v}}}: \mu\left(\sigma^{i_{r}+1}(\mathbf{w})\right)>0\right\rangle\right) \subset Y_{a, Q} \cap D_{+}\left(y_{\mathbf{v}}\right) .
$$

Hence, $Y_{a, Q} \cap D_{+}\left(y_{\mathbf{v}}\right)$ is a dense open subset of $S_{i_{0}} \cup S_{i_{1}} \cup \cdots \cup S_{i_{\ell}}$. Since the open subvarieties $\left\{D_{+}\left(y_{\mathbf{v}}\right) \cap Y_{a, Q}: \mathbf{v}\right.$ well-spaced $\}$ cover $Y_{a, Q}$, this completes the proof.

\section{A $K / k$ twist of $Y_{a}$}

Let $K / k$ be a cyclic Galois extension of degree $n$, and let $R_{K}=R \otimes_{k} K$. Fix a basis $\left\{\alpha_{0}, \ldots, \alpha_{n-1}\right\}$ of $K$ as a $k$-vector space, as well as a generator $\tau$ of $\operatorname{Gal}(K / k)$.

Let $T$ be a set of representatives for the orbits of $\mathcal{V}_{n}$ under the action of the shift operator $\sigma$. Consider the $K$-isomorphism $\psi: \operatorname{Proj} K\left[\left\{z_{\mathbf{v}}: \mathbf{v} \in \mathcal{V}_{n}\right\}\right] \rightarrow \operatorname{Proj} K\left[\left\{y_{\mathbf{v}}\right.\right.$ : $\left.\left.\mathbf{v} \in \mathcal{V}_{n}\right\}\right]$ determined by

$$
\begin{aligned}
y_{\mathbf{v}} & \mapsto \alpha_{0} z_{\mathbf{v}}+\alpha_{1} z_{\sigma(\mathbf{v})}+\cdots+\alpha_{n-1} z_{\sigma^{n-1}(\mathbf{v})} \quad \text { for } \quad \mathbf{v} \in T, \\
y_{\sigma^{i}(\mathbf{v})} & \mapsto \sum_{j=0}^{n-1} \tau^{i}\left(\alpha_{j}\right) z_{\sigma^{j}(\mathbf{v})} \quad \text { for } \quad \mathbf{v} \in T, \quad i=1, \ldots, n-1 .
\end{aligned}
$$

Define $X_{K, a}^{0}=\psi_{R_{K}}^{-1}\left(Y_{a}\right)$. Abusing notation, we write $\tau$ for the endomorphism of Proj $R\left[\left\{z_{\mathbf{v}}: \mathbf{v} \in \mathcal{V}_{n}\right\}\right] \times{ }_{R} R_{K}$ given by id $\times \tau$. Let $\phi: \mathbb{P}_{R}^{N} \rightarrow \mathbb{P}_{R}^{N}$ be the automorphism of Lemma 3.1. The following diagram commutes:

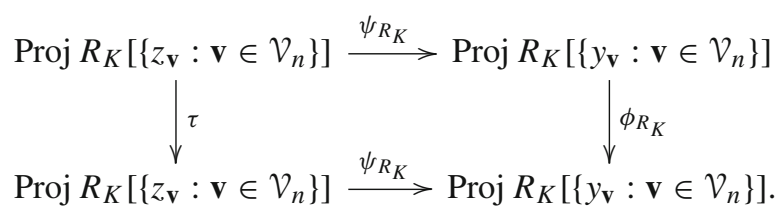

By Lemma 3.1, the map $\phi$ preserves $Y_{a}$. Together with the commutativity of the above diagram, this implies that $X_{K, a}^{0}$ descends to an $R$-scheme.

\section{Proof of Theorem 1.1}

Let $K / k$ be a cyclic Galois extension of degree $n$ and let $P(x) \in k[x]$ be a separable polynomial of degree $d n$ or $d n-1$ for some $d$.

Lemma 5.1 There exists a smooth projective variety $X=X_{K / k, P(x)} \rightarrow \mathbb{P}_{k}^{1}$ such that $X_{\mathbb{A}^{1}} \cong X_{K, P(x)}^{0}$ and that $X_{\mathbb{P}^{1} \backslash\{0\}} \cong X_{K, P\left(1 / x^{\prime}\right) x^{\prime d n}}^{0}$, where $x^{\prime}=1 / x$.

Proof We will construct $X$ by glueing $Y_{P(x)}$ and $Y_{P\left(1 / x^{\prime}\right) x^{\prime d n}}$ over Spec $k\left[x^{ \pm 1}\right]$ and Spec $k\left[x^{\prime \pm 1}\right]$, in a way which is compatible with the map $\psi$ from Sect. 4. Let $y_{\mathbf{v}}$ 
denote the coordinates on $Y_{P(x)}$ and let $y_{\mathbf{v}}^{\prime}$ denote the coordinates on $Y_{P\left(1 / x^{\prime}\right) x^{\prime d n}}$. By Lemma 2.1 (b), the morphism

$$
Y_{P\left(1 / x^{\prime}\right) x^{\prime d n}} \times_{\mathbb{A}^{1}} \operatorname{Spec} k\left[x^{\prime}, x^{\prime-1}\right] \rightarrow Y_{P(x)} \times_{\mathbb{A}^{1}} \operatorname{Spec} k\left[x, x^{-1}\right]
$$

where $y_{\mathbf{v}} \mapsto\left(x^{\prime}\right)^{d \lambda(\mathbf{v})} y_{\mathbf{v}}^{\prime}$ and $x \mapsto 1 / x^{\prime}$ is well-defined and is an isomorphism. Since $\lambda(\mathbf{v})=\lambda(\sigma(\mathbf{v}))$, this morphism is compatible with $\psi$ and thus gives a glueing of $X_{K, P(x)}^{0}$ and $X_{K, P\left(1 / x^{\prime}\right) x^{\prime d n}}^{0}$.

Proposition 5.2 The variety $X$ is a smooth proper compactification of $X_{0}$, the generic fiber of $X \rightarrow \mathbb{P}^{1}$ is a Severi-Brauer variety, and the degenerate fibers of $X \rightarrow \mathbb{P}^{1}$ lie over $V\left(P\left(x_{0} / x_{1}\right) x_{1}^{d n}\right)$ and consist of the union of $n$ rational varieties all conjugate under $\operatorname{Gal}(K / k)$.

Proof The compatibility (2) together with Proposition 3.3 and Corollary 3.4 implies that

$$
\left(X \times_{\mathbb{P}^{1}} \mathbb{A}^{1}\right) \cap D_{+}\left(z_{(1,1, \ldots, 1)}\right) \cong X_{0}
$$

which gives the first claim. The second claim is immediate from the construction of $X$, and the third claim follows from Proposition 3.5 and the compatibility (2).

Proposition 5.2 completes the proof of Theorem 1.1 .

\section{References}

1. Artin, M.: Left ideals in maximal orders. In: Brauer Groups in Ring Theory and Algebraic Geometry (Wilrijk, 1981). Lecture Notes in Mathematics, vol. 917, pp. 182-193. Springer, Berlin (1982)

2. Colliot-Thélène, J.-L., Harari, D., Skorobogatov, A.N.: Valeurs d'un polynôme à une variable représentées par une norme. In: Number Theory and Algebraic Geometry. London Mathematical Society Lecture Note Series, vol. 303, pp. 69-89. Cambridge University Press, Cambridge (2003)

3. Colliot-Thélène, J.-L., Van Geel, J.: Le complémentaire des puissances $n$-ièmes dans un corps de nombres est un ensemble diophantien (2014). arXiv:1401.0915

4. Derenthal, U., Smeets, A., Wei, D.: Universal torsors and values of quadratic polynomials represented by norms. Math. Ann. doi:10.1007/s00208-014-1106-7

5. Harris, J.: Algebraic Geometry. Graduate Texts in Mathematics, vol. 133. Springer, New York (1992)

6. Kang, M.C.: Constructions of Brauer-Severi varieties and norm hypersurfaces. Can. J. Math. 42(2), 230-238 (1990)

7. Poonen, B.: The set of nonsquares in a number field is diophantine. Math. Res. Lett. 16(1), 165-170 (2009)

8. Várilly-Alvarado, A., Viray, B.: Higher-dimensional analogs of Châtelet surfaces. Bull. Lond. Math. Soc. 44(1), 125-135 (2012) 